\title{
Direct Detection Carbon Electron Energy Loss Spectroscopy on Zeolites
}

\author{
M. Cem Akatay ${ }^{1}$ and Steven A. Bradley ${ }^{1}$ \\ ${ }^{1 .}$ UOP LLC, A Honeywell Company
}

Chemical analysis at the nanoscale is of vital importance in nanomaterials research and development. Recent advances in aberration correction of the transmission electron microscopes coupled with improvements in electron spectrometers paved the way for high-resolution chemical analysis of nanostructures by aberration corrected STEM and parallel EELS [1-2]. The integration of direct electron detectors with energy loss spectroscopy further increased the sensitivity of electron spectroscopy [3-4]. These advances enabled high resolution chemical analysis of materials at a much lower dose with minimal or negligible alteration of the materials chemistry by the electron beam. Such a capability offers a great opportunity for the chemical analysis of materials where both the structure and the chemistry of the materials would alter under high electron dose needed for spectroscopic analysis.

In this paper, Quantum Gatan Imaging Filter coupled with direct electron detector (K2) is used to collect electron energy loss spectra (EELS) in the electron counting mode to demonstrate the use of direct electron detection EELS on a beam sensitive material. The material of choice was zeolite and the goal was to visualize and chemically differentiate carbonaceous species on this zeolitic material. Zeolites are crystalline aluminosilicates that are used as industrial adsorbents and catalysts thanks to their high surface area and pore network of well-defined cages and channels [5]. The focus of this work is on a zeolite embedded with metal nanoparticles that serves as a bifunctional catalyst for hydrocarbon chemistry [6]. During the catalytic reaction carbonaceous species deposit on the material. These carbon species are regarded as coke. Coke is detrimental to the performance of the catalyst as it hinders diffusion and accessibility of active sites. [7]. The goal of this work was to understand the location of these coke species on the catalyst and the chemical nature of the coke.

The EELS data is collected at multiple microscopes operating in STEM mode at $200 \mathrm{kV}$. The material was noble metal supported on a zeolite. The catalysts were spent resulting in 1-3 wt. \% carbon. Carbon energy loss spectra were collected both with direct electron detector (K2) as well as the conventional indirect electron detector (CCD). STEM-EELS is employed to map the carbon on the zeolite. The beam dose is kept low to avoid structural damage to the zeolite by radiation. Under these conditions, CCD coupled EELS failed to visualize the coke at low levels of coke $(<1 \mathrm{wt}$. \%) whereas K2 coupled EELS succeeded in mapping carbonaceous species even at these low levels. Coke was found within the zeolite channels and is associated with both the zeolite and metal particles. These two sites exhibited different characteristics on their carbon EELS spectra with respect to the strength of the pi* peak which is associated with the graphitic carbon as opposed to amorphous carbon where such peak is absent [8]. Graphitic coke was associated with metal particles whereas amorphous coke was found on the zeolitic sites. The characteristic feature for graphitic carbon was highly beam sensitive and graphitic carbon was readily amorphized under electron beam. As a result, CCD coupled EELS failed to detect these species and they were discovered only by the K2 coupled EELS.

In summary, analyzing carbonaceous species on zeolitic materials by electron microscopy is a challenging task due to highly beam sensitive nature of both the zeolite and the carbon. Under electron beam the zeolitic structure can get destroyed and the chemical nature of the carbonaceous species may 
alter. Direct Detection EELS helped to overcome these challenges and enabled the detection of low concentration coke on the zeolites, as well as helped to differentiate between beam sensitive species of carbon.

\section{References:}

[1] DA Muller et al, Science 319 (2008), p. 1073.

[2] N. Biškup et al, Phys. Rev. Lett. 112, p. 087202.

[3] RS Ruskin, Z Yu, and N Grigorieff, J. Struct. Biol. 184 (2013), p. 385.

[4] JL Hart et al, Microsc. Microanal. 22 (S3) (2016), p. 336.

[5] R. Broach and S. Kulprathipanja in "Mixed-Matrix Membranes, in Zeolites in Industrial Separation and Catalysis", ed S. Kulprathipanja, (Wiley-VCH).

[6] V. Kanazirev J. Chem. Soc. Chem. Communications (1990), p. 712.

[7] S.I. Sanchez, M.D. Moser, S.A. Bradley, ACS Catal. 4 (2014), p. 220.

[8] S. Bare et al, ACS Catalysis 7 (2017), p. 1452.

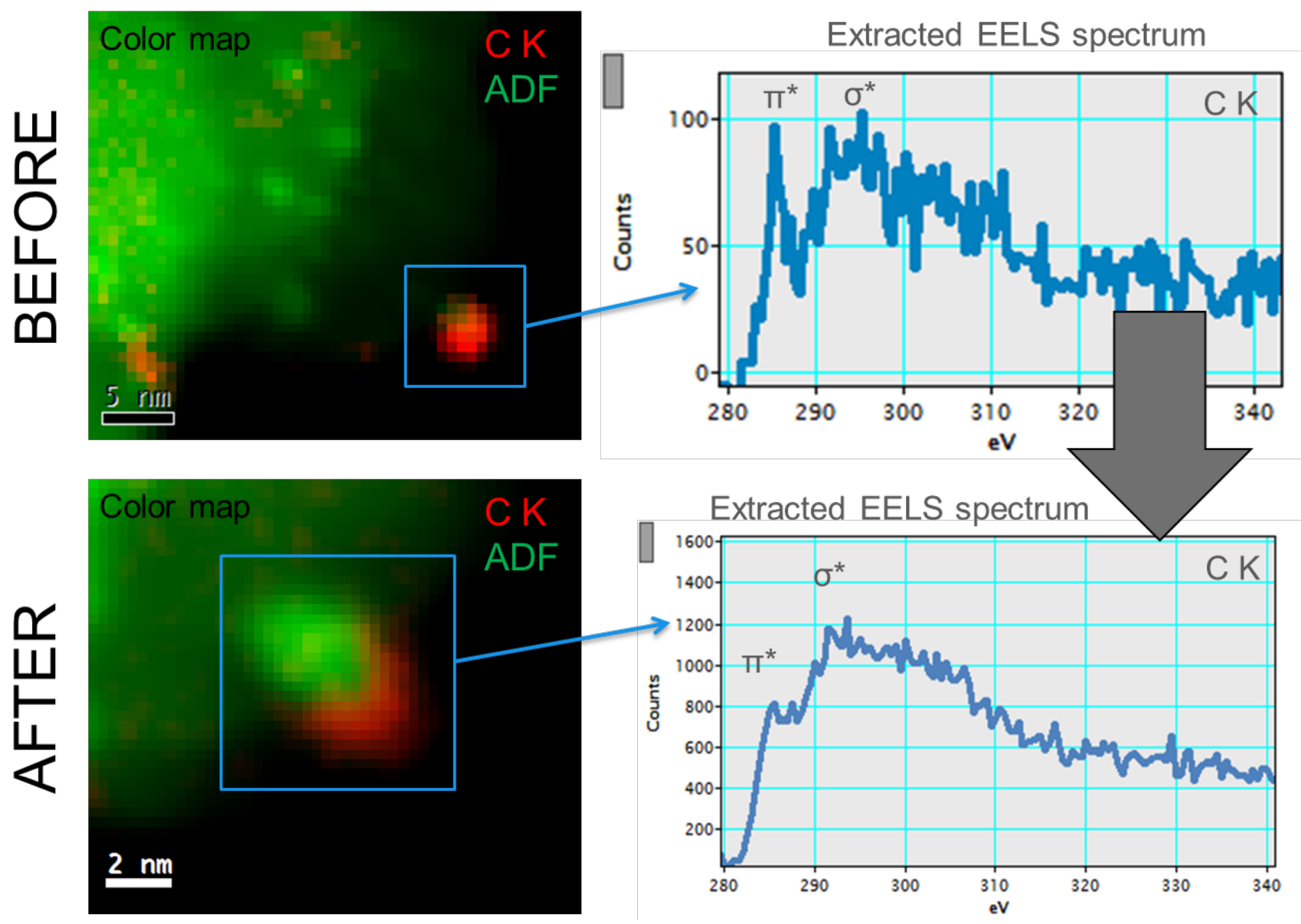

Figure 1. (BEFORE) Composite EELS map showing the ADF signal in green and the carbon signal in red. The corresponding EELS signal exhibits well-pronounced pi*-peak and is highly graphitic in nature (AFTER) Same region after electron beam exposure. The pi*-peak has disappeared and mostly amorphous in nature. 